\title{
Therapeutics Delivery Behind, Through and Beyond the Blood Brain Barrier
}

\author{
Alain L Fymat* \\ International Institute of Medicine and Science, USA \\ Submission: July 02, 2017; Published: July 26, 2017 \\ *Corresponding author: Alain L Fymat, International Institute of Medicine and Science, California, USA, Tel: (760) 485-9149; \\ Email: alain.fymat@fiimas.org
}

\begin{abstract}
The delivery of therapeutics through, behind and beyond the blood brain barrier (BBB) is a subject of current intensive research. A witness to this statement is the number of published research articles and national/international conferences on the subject. This is understandable because of the multiplicity (about 400) of brain diseases, although some of them are cognizant disorders. Some of these disorders cause different effects on the BBB from disrupting it, breaking it down, opening it, or damaging it, while other disorders may still have unknown effects on it. It is therefore of utmost importance to grasp the difficulties encountered when attempting to deliver therapeutics at the right brain locations and at the right time-dose fractionations. For this purpose, I will first briefly review the brain diseases and their effects on the BBB, discuss the early discovery and physiological development of the BBB, provide a brief primer on nanoneurology, and discuss the clinical significance of the BBB as a drug target. I will then analyze the drug delivery difficulties and their limitations, the drug targeting mechanisms ("behind", "through" and "beyond" the BBB), and describe the novel uses offered by nanotechnology (nanoparticles and nanodevices). I will then conclude with future prospects in this research field.
\end{abstract}

Abbreviations: BBB: Blood Brain Barrier; BPB: Brain Protective Barriers; B[iCSF]B: Brain inner CSF barrier; B[oCSF]B; Brain outer CSF barrier; BRB: Brain Retinal Barrier; CNS: Central Nervous System; CSF: CerebroSpinal Fluid; FDA: (U.S.) Food \& Drug Administration; GBM: Glioblastoma; HDCA: Hexa Decyl Cyano Acrylate; H3M: 3-Helix Micelles; HIFU: High-Intensity Focused Ultrasound; LDLRP/Epic: Low Density Lipoproteins/ Related Protein with Engineered Peptide Compound; MEN: Magneto-Electric Nanoparticles; ND: Nanodevices (ND); NN: Nanoneurology; NP: Nanoparticles; PACA: Poly alkyl CyanoAcrylate; PLGA: Poly LacticCo Glycolic Acid; PMA: Peptidomimetic Monoclonal Antibodies; RDNA (recombinant DNA); RES: Reticulo-Endothelial System

Drugs Cited: Bradkynin; Camphotothericin; Casomorphion; Dalagrin; Daunomycin; Doxorubicin; Insulin; Low-Density Lipoprotein/Related Protein with Engineered Peptide Compound; Mannitol; Peptidomimetic Monoclonal Antibodies

\section{Brain Protective Barriers}

The brain has five protective barriers (BPB) that hinder the delivery of therapeutic drugs. They describe the five main interfaces between the central nervous system (CNS) and the periphery. These include: the blood brain barrier (BBB) that extends down the spinal cord; the brain cerebrospinal fluid (CSF) barrier (B[CSF]B]; the brain-inner CSF barrier (B[iCSF] b); the brain-outer CSF barrier $\mathrm{B}[\mathrm{oCSF}] \mathrm{B}$; and the brain retinal barrier $(\mathrm{B}[\mathrm{R}] \mathrm{B})$. We shall mostly be concerned with the $\mathrm{BBB}$ and use the denomination BBB as if it applied (though erroneously) to all BPBs.

All interfaces are formed by tight junctions. In the case of the $\mathrm{BBB}$, the tight junctions are between the endothelial cells of the primary vasculature with primary manifestation being the impermeability of the capillary wall due to the presence of the junctions and a low endocytic activity. For B [CSF] B, the tight junctions are between the epithelial cells of the choroid plexus. In the case of the $\mathrm{B}[\mathrm{iCSF}] \mathrm{B}$, the junctions are between the neuroependymal cells lining the ventricular surfaces. As for B [oCSF] $B$, the junctions are between endothelial cells of the arachnoid vessels (the pia arachnoid). The BPBs hinder the delivery of most pharmaceuticals (diagnostic, therapeutic agents) to the brain.

\section{a. Some brain diseases and their effects on the Blood Brain Barrier}

There are approximately 400 neural disorders some which being cognitive. Table 1 below shows only some of these, specifically those due to a failure of the BBB as a result of disruption, breakdown, opening, failure, damage (inflammatory) and increased permeability, but also some diseases for which the effect on the BBB is still unknown.. Neurological disorders are 
caused by inflammation, tumors or degenerative disorders. With the growing aging population, many such neurodegenerative disorders, cancers and infections of the brain will become more prevalent. Of interest to us are those disorders that require treatment by delivery of therapeutic drugs across the BBB.

Table 1: Some brain diseases and their corresponding effects on the BBB.

\begin{tabular}{|c|c|c|c|}
\hline Disease & BBB effect & Disease & BBB effect \\
\hline Alzheimer & Disruption/ breakdown & $\begin{array}{c}\text { Multiple sclerosis (immune } \\
\text { system deficiency) }\end{array}$ & Breakdown \\
\hline Brain abscess & Unknown mechanism & $\begin{array}{c}\text { Neuromyelitis optica (Devic's } \\
\text { disease) }\end{array}$ & Breakdown \\
\hline Cerebral edema & Opening (due to hypoxia) & $\begin{array}{c}\text { Prion and prion-like diseases } \\
\text { (Parkinson, Alzheimer) }\end{array}$ & Unknown penetration mechanism \\
\hline De vivo & Unknown mechanism & $\begin{array}{c}\text { Progressive multi-focal leuko- } \\
\text { encephalopathy }\end{array}$ & Disruption \\
\hline Epilepsy & Disruption/failure & Rabies & Increased permeability \\
\hline HIV encephalitis & Damage (inflammatory) & $\begin{array}{c}\text { Systemic inflammation (sterile, } \\
\text { infectious) }\end{array}$ & Disruption? \\
\hline (latent HIV crosses the BBB) & Disruption & Tripanosomasis (sleep thickness) & Disruption \\
\hline Meningitis & &
\end{tabular}

\section{b. Early Discovery and Physiological Development}

The existence of compartmentalization within the brain was discovered early in the lifetime of individuals. It was then called "hematoencephalic barrier". It was evidenced from tissue staining both outside (Ehrlich) and inside the brain. Observation and proof of its existence was further obtained from electron scanning microscopy (early 1960s). The early hematoencephalic barrier was shown by Lewandowsky in the early 1990s. That the BPBs are operative at birth was confirmed by uptake measurements of several chemicals that showed no differences between newborns and adults. Table 2 lists some of the various chemicals used in the brain uptake measurements: Please note that in cancer studies, newborns and children should not be considered as small adults as they are still growing and developing in ways different from adults.

Table 2: Chemicals Used in Uptake Measurements Showing No Differences between Newborns and Adults.

\begin{tabular}{|c|c|}
\hline Chemicals & Acetamide \\
\hline Alcohol & Benzyl Alcohol \\
\hline Caffeine & Cytosine \\
\hline Ethanol & Ethylene Glycol \\
\hline Glycol & Heroin \\
\hline Mannitol & Methanol \\
\hline Phenobarbitol & Phenytoninpropylene \\
\hline Thiourea & Urea \\
\hline
\end{tabular}

\section{A Brief Primer on Nanoneurology}

\section{A. Definitions}

Neurology deals with the diagnosis and treatment of the central nervous system (brain and spinal cord). Nanoneurology (NN) is neurology at the nanoscale employing nanoparticles (NP) and nanodevices (ND) for imaging, diagnosis and treatment at a finer spatial scale and that may additionally reach certain tissues or organ tissues that were previously unreachable by conventional approaches.

\section{B. Anatomy of the blood brain barrier}

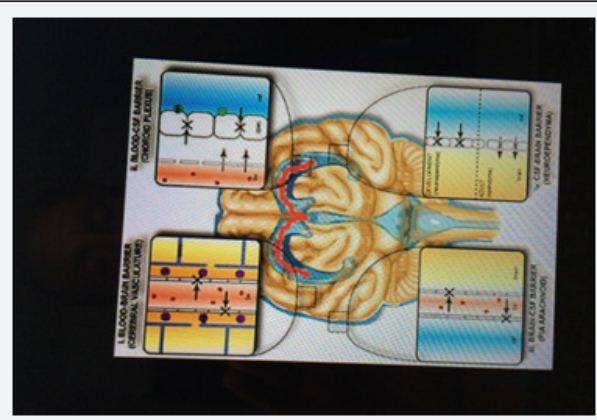

Figure 1: Axial view of the brain protective barriers.

The BBB is formed by the brain endothelial cells, which are the flat cells lining the capillaries of the blood and lymphatic vessels. The astrocytes, which are large neuroglia cells of the nervous tissue are necessary to create the BBB. The capillaries are connected by tight junctions around them. These junctions do not exist in the normal circulation. The BBB is a highly selective, semi-permeable membrane barrier separating the circulating blood from the brain extra-cellular fluid. A few brain regions do not have a BBB. These are the circumventricular organs, the roofs of the third and fourth ventricles, the roof of the diencephalon, and the pineal gland. Figures 1-4 describe the brain anatomy of interest. Figure 2 is a sketch of the left lateral view of the brain structural areas (in purple color: the frontal lobe and the precentral gyros; in yellow color, the central fissure, the post-central gyrus, and the parietal lobe; in green color: the parieto-occipital fissure (deep); in blue color: the temporal lobe and the lateral fissure; and in the uncolored areas are the pons, medulla, the spinal cord, and the cerebellum). 

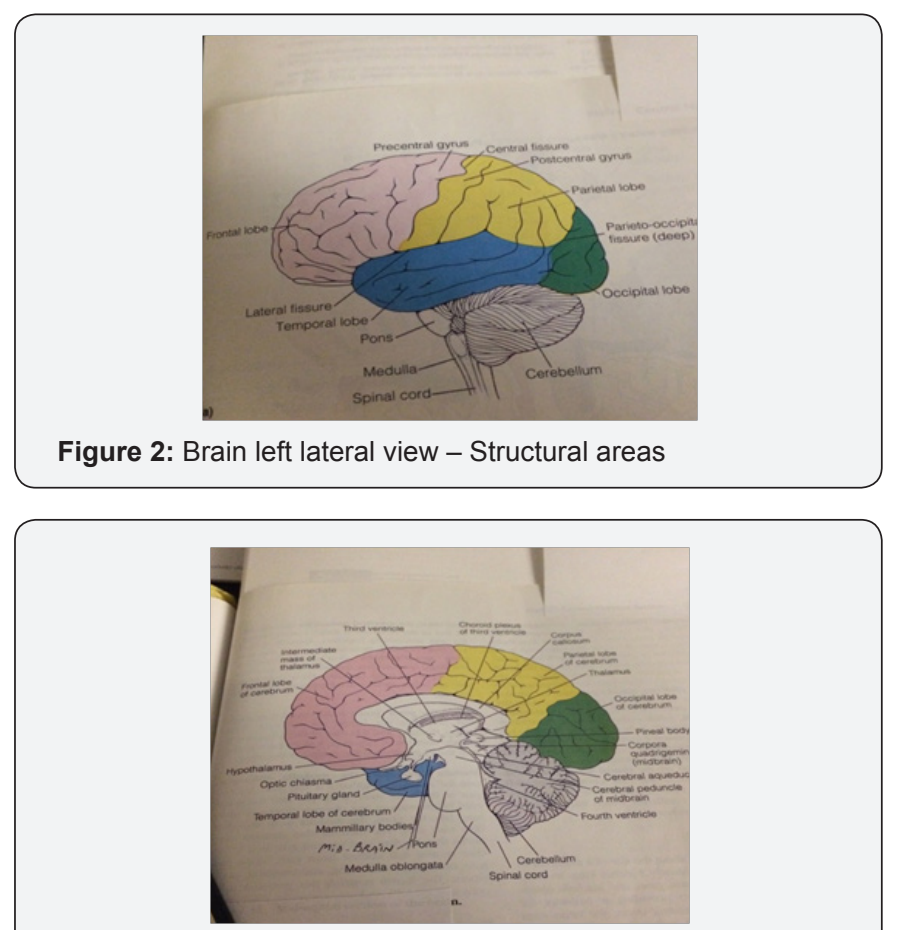

Figure 3: Brain mid sagittal section.

Figure 3 is a brain mid-sagittal section (in purple color: the frontal lobe of the cerebrum; in yellow color, the parietal lobe of the cerebrum; in green color: the parieto-occipital lobe; in blue color: the temporal lobe; in grey color: the cerebellum; and in the uncolored areas viewed clockwise are: upper: the intermediate mass of the thalamus, the third ventricle (whose roof is devoid of BBB), the choroid plexus, the corpus callosum, and the thalamus; center: mamillary bodies, pituitary gland, optic miasma, hypothalamus, pineal gland (devoid of BBB), corpora quadrigemine (midbrain), cerebral aqueduct; and lower: medulla oblongata, pons, cerebral peduncle of mid-brain, fourth ventricle (whose roof is devoid of BBB). Lastly, Figure 4 describes the ventricles and the CSF. It is a three-dimensional lateral view showing clockwise from the pituitary gland: the pituitary gland, the third ventricle (whose roof is devoid of BBB), the corpus callosum, the cerebrum covered with pia mater, the choroid plexus, the cerebral vein, the superior sagittal sinus, the subarachnoid space, the arachnoid vili, the arachnoid, the meningeal dura mater, the periosteum dura mater, the fourth ventricle (whose roof is devoid of $\mathrm{BBB}$ ), and the median aperture.

\section{Physiology of the blood brain barrier}

In its integral form, the BBB is a selective filter that:

a) Allows the passage of essential nutrients, water, some gases, lipid-soluble molecules, and hydrophobic molecules $\left(\mathrm{O}_{2}, \mathrm{CO}_{2}\right.$, hormones $)$

b) Allows the transport of metabolic products to the brain (glucose with specific proteins) c) Restricts the diffusion of microscopic objects (e.g. bacteria) and large hydrophilic molecules.

d) Prevents the entry of polar and lipid-insoluble substances, and lipophilic neurotoxins.

Entry into the BBB is mediated by P-glycoproteins. It may be disrupted by brain tumors and other pathologies. The passage mechanism is one of passive diffusion combined with selective molecular transport of glucose and amino-acids, among other substances. Figure 4 shows the distribution of cranial nerves (in blue color: the sensory nerves; in red color: the motor nerves).

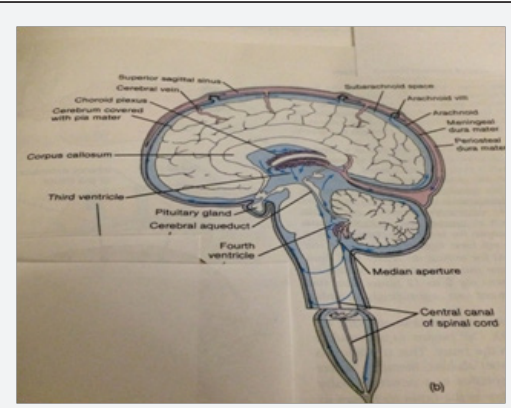

Figure 4: Three-dimensional view of the left ventricles and the cerebro-spinal fluid.

\section{Function of the blood brain barrier}

The barrier is a very effective protector of the brain from most pathogens. Blood-borne brain infections are very rare but could be very serious and difficult to treat. Antibodies are too large to cross it. The barrier behaves as a continuous lipid layer, which is a major obstacle preventing the passage of polar and lipid insoluble substances and drugs. Figure 5 illustrates the distribution of cranial nerves (sensory and motor) and Figure 6 is a left lateral view of the brain functional areas. In purple color: very light: the pre-motor area; light: the primary motor area; medium: the frontal association area (intellectual and psychic functions, and language); and dark: the motor area (speech). In yellow color: light: the somatic sensory area; and dark: speech and reading. In blue color: light: the memory and olfactory area; medium: not specified; and dark: the auditory area). In green color: the visual area [1].

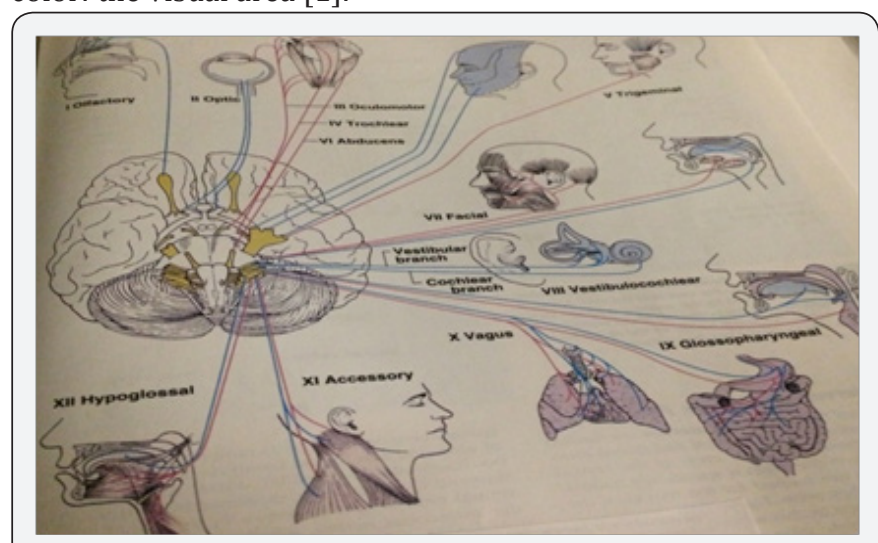

Figure 5: Distribution of cranial nerves. 


\section{Clinical Significance for Drug Targeting}

The BBB excludes from the brain: $100 \%$ of large molecule neuro-therapeutics and more than $98 \%$ of all small drugs (including many important diagnostic and therapeutic agents). Only certain antibiotics are able to pass $[2,3]$. The BBB is not always intact in tumors because vascular endothelial cells and associated pericytes are not intact. The basement membrane is also incomplete. Astrocytes may contribute to resistance of brain tumors to therapy. The barrier becomes more permeable during inflammation allowing some antibiotics and phagocytes to pass.

A. Drug delivery hindrance: In its protective role, the BBB hinders the delivery of many potentially important diagnostic and therapeutic molecules and antibodies, which are otherwise effective in diagnosis and therapy.

B. Passive diffusion through endothelial cells and endogenous transporters: This depends on their lipophilicity, degree of ionization, molecular weight, relative brain tissue, and plasma bindings.

C. Competition on transporters with endogenous products or nutrients: Can occur and limit drug transfer

D. Drug delivery strategies: These may involve modifying the drug itself, coupling the drug to a vector for receptor-mediated or absorption-mediated transcytosis. Such strategies are needed to safely and effectively deliver therapeutic molecules,

\section{Drug Targeting Difficulties}

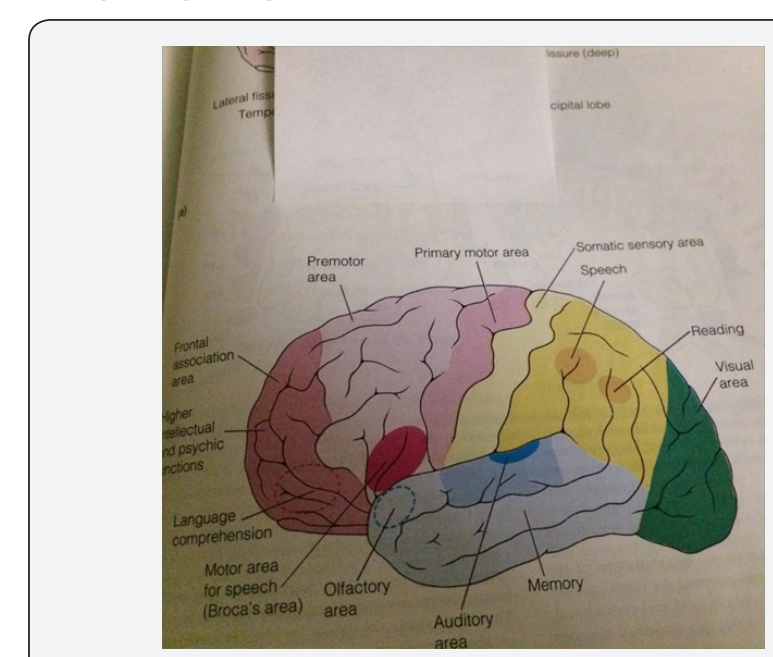

Figure 6: Left lateral view of the brain functional areas.

BBB is the major impediment and main limitation for the treatment of neurological disorders. The reason is the close intercellular contact between cerebral endothelial cells due to tight junctions that prevents passive diffusion of hydrophilic components from the blood stream into the brain. Many drugs are unable to reach the brain at therapeutic concentrations. The complex microenvironment of tumors presents a challenge in developing effective anti-cancer treatments that attempt to harness nanotechnology as illustrated in the case of tumors generally, brain tumors particularly. The left side of the Figure illustrates tumorigenesis wherein the tumor develops its own blood supply, whereas on the right side, the tumor develops its own lymphatic drainage system. [As a reminder, the stroma is the framework, usually of connective tissue, of an organ, gland or other structure (tumor). It is to be distinguished from the parenchyma or specific substance of the part supported by the stroma] (Figure 7).

\section{Attempts To Overcome the Limitations of the Blood Brain Barrier}

Overcoming the difficulty of delivering therapeutic agents to specific regions of the brain presents a major challenge to the treatment of most brain disorders. The following approaches are being explored:

I. Chemical modification

II. Hydrophobic analogs development

III. Active compound linking to a specific carrier

IV. Transient opening of the BBB: It can be achieved via intra-carotid infusion of bradkynin analogs or hypertonic mannitol solutions (mannitol is a hexahydric alcohol driven by reduction of fructose used in renal function testing to measure glomerular filtration and intravenously as an osmotic diuretic);

V. P-glycoprotein (P-Gp) modulation: P-Gp substrates are actively pumped out of the cell and into the capillary lumen. Many P-Gp are available to enhance the therapeutic effects of peripherally active drugs [4].

\section{Drug Targeting Mechanisms}

There are essentially three mechanisms for drug targeting in the brain:

i. "Through/across" the brain (disrupts the BBB).

ii. "Behind" the brain (bypasses the BBB).

iii. Targeting of brain regions or tumors beyond the BBB.

\section{A. Drug targeting through the blood brain barrier}

The approach consists in disrupting the BBB by modifying the tight junctions and causing controlled and transient increases in the permeability of the brain capillaries. This is accomplished by:

i. Molecular Trojan horses: These are interference drugs across the BBB, e.g., PMA (Peptidomimetic Monoclonal Antibodies) and RDNA (recombinant DNA). PMAs transport large molecules, e.g., antibodies, recombinant proteins, and nonviral gene medicines). 
ii. Particulate drug carrier systems: They enhance delivery of therapeutic molecules across the CNS vascular barrier to achieve high drug concentration. There is an intensive search for alternative routes, including intra-nasal delivery systems; convection-enhanced diffusion systems; and intrathecal/intraventricular systems. The process is one of osmosis [5], which can be accomplished biochemically by the use of vasoactive substances (e.g. bradkynin [6]) or even exposure to high-intensity focused ultrasound (HIFU).

There are various efflux transporters that are expressed on cerebral endothelial cells that are

I. Implicated in the delivery to the brain of nutrients, ions and vitamins; and

II. Extrude endogenous substances or xenobiotics out of the brain and into the general circulation. (Cytosis is a condition in which there is more than the usual number of cells. In transcytosis, the cells remain trapped in the endothelial cells of the capillaries.)

The receptor-mediated transport systems enable molecules to cross the BBB in vivo. Polymers, including biodegradable polymers, are used for malignant tumors and Alzheimer.

\section{B. Drug targeting behind the blood brain barrier}

There are essentially three processes:

i. Intranasal: For example, for insulin delivery, intranasal injection shows poor intake whereas peripheral injection may provoke hypoglycemia.

ii. Intracranial implantation (such as with needles): In rats, small amounts of pancreatic islets increase brain insulin content and attenuate cognitive dysfunctions without altering peripheral glucose homeostasis.

iii. Convection-enhanced distribution.

Nonetheless, the challenge remains to develop drug delivery strategies that allow the passage of drug molecules through or behind the BBB in a safe and effective manner.

\section{Approaches to Enhancing Therapeutics Delivery}

We distinguish between three different approaches:

\section{A. Physiological approaches}

The development of new drugs against CNS disorders has not kept pace with progress in molecular neurosciences. Thus, new drugs discovered are unable to cross the BBB, the culprit being the lack of appropriate delivery systems. Further, localized and controlled delivery of the drugs at the desired sites is preferred because it reduces toxicity and increases treatment efficiency/ efficacy. Here, LDLRP/Epic, a low density lipoprotein/related protein with engineered peptide compound, is a new effective therapeutics. It improves the transcytosis capacity of specific receptors expressed across the BBB (Figure 7).

\section{B. Chemical delivery systems}

These delivery systems include:

i. $\quad$ Lipid-mediated transport.

ii. Pro-drug approach.

iii. Lock-in systems.

\section{Biological delivery}

The pharmaceuticals are re-engineered to cross the BBB via specific endogenous transporters located within the brain capillary endothelium.

\section{Other drug delivery systems}

I. Receptor-mediated transport systems that enable drug molecules to cross the BBB in vivo. Such systems exist for certain endogenous peptides (insulin, transferin).
II. Solid lipids.
III. Polymeric.
IV. Mesoporous silica, and
V. Inorganic.

\section{Use of Nanotechnology}

One of the most promising applications of nanotechnology is in clinical neuroscience and multiple tasks can be carried in a pre-defined sequence [7-15]. Figure 7 illustrates the action of nanoparticles against cancer. Figure 7 the layered NP consists of three components:

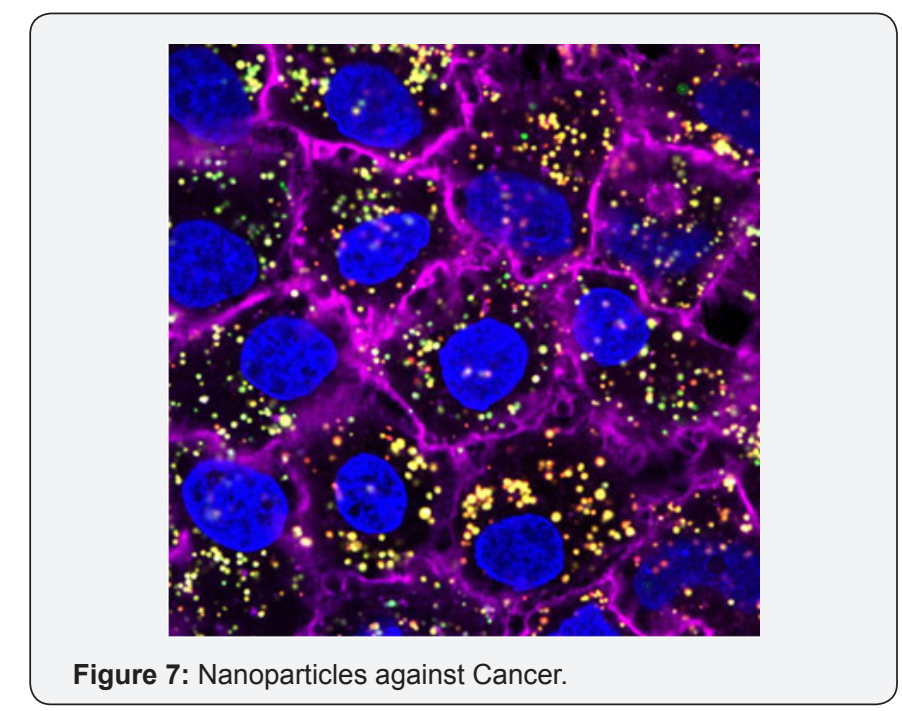

I. A core vesicle with a double-layered membrane. It is filled with water and hydrophilic and/or hydrophobic drugs;

II. A multi-layered shell; and an exterior shell that targets the NPs to cancer cells and prevents them from being detected by the immune system. The purposes of a multi-layered shell are: to stabilize the NPs; prevent drug leakage; target the NPs 
to the slightly acidic environment of the tumor; minimize the interactions of the NPs with non-cancerous cells; and pass unnoticed by the immune system. The multi-layered NP can also transport drugs that are not easily stored in the core (e.g., highly charged nucleic acids). These molecules can be separated from drugs in the core that could inactivate their therapeutic effects (e.g., plasma drugs).

Several nanocarriers have been developed for drug delivery at the right address. However, challenges still remain, including: How not to let the medicine (s) act before they reach the right place. Carriers usually encapsulate drugs through long-range electrostatic interactions wherein the carrier attracts oppositelycharged medicines. Other tools are available to trigger the release of drugs, e.g. magnetic fields, different ph-values, etc., but, in each case, the problem of efficiency of the drug release remains. Nonetheless, work is still needed to determine the most effective NTs for brain tumors.

\section{A. Nanoparticles}

The various NPs include:

i. Microspheres.

ii. Bionanocapsules.

iii. Radiolabeled polyethylene glycol-coated: Hexa Decyl Cyano Acrylate; (HDCA); PolyAlkylCyanoAcrylate (PACA); PolyLacticCoGlycolic Acid (PLGA); Peptidomimetic Monoclonal Antibodies; (PMA).

iv. Magneto-Electric Nanoparticles (MENs) for targeted delivery and drug release across the BBB + wireless stimulation of cells deep in the brain.

v. Bioavailability-improved nanoscale particles and molecules: Nanoscale particles and molecules can also be developed to improve drug bioavailability, i.e., the presence of drug molecules where they are needed in the body and where they will do the most good. Drug delivery focuses on maximizing bioavailability both at specific places in the body and over a period of time. It can be achieved by molecular targeting by nano-engineered devices targeting the molecules and delivering drugs with cell precision. The basic process to use drug delivery involves at least three steps:

a) Encapsulation of the drugs.

b) Successful delivery of said drugs to the targeted region of the body, and

c). Successful release of that drug there. Several NPs are employed,

vi. Nanogels: They were previously discarded because they stick together with their neighbors (lost colloidal stability) when trying to "upload" the drugs within them. This made delivery impossible or ineffective. However, a solution to the stickiness was developed by Prof. Potemkin at the University of Florida. Figure 6 is an illustration of multi-shell nanogels with responsive shell permeability figure 8 .

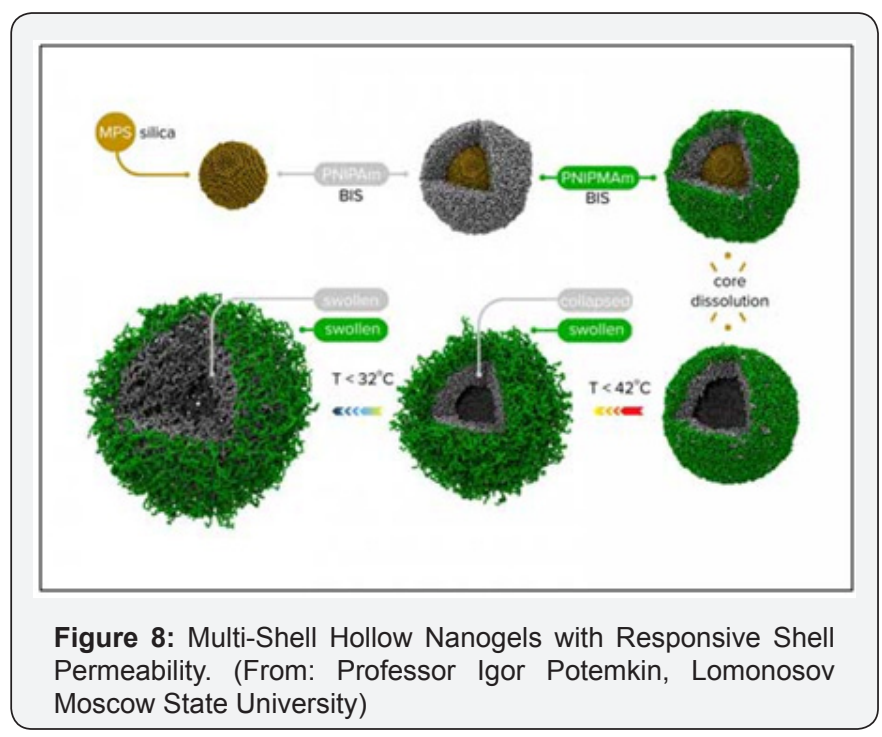

Potemkin solved the nanogel problem by creating a carrier surrounded by 2 "membranes" (or shells) of different chemical structure around a silica core. At the end of the synthesis, the core will be chemically dissolved leaving only the "empty" cavity. The "outer"porous shell plays a protective (stabilizing) role and hinders aggregation of nano-capsules, while the pores of the "inner" shell can open and close depending on the temperature due to variable interactions between its monomeric units. At the time of filling, the pores of both shells are "open" and the nanogel absorbs the drug molecules as a sponge. Then, the temperature changes and the pores of the inner shell close locking-in the cavity and readying the drug for delivery. Subsequently, the pores will open again and the guest molecules will be released only in the places where the temperature allows. Nonetheless, work is still needed to demonstrate that nanogels are the ideal drug delivery carrier.

Other available technologies include:

a. Liposomes

b. Peptides: A peptide is a compound of 2 or more amino acids in which the alpha carboxyl group of one is united with the alpha amino group of the other with the elimination of a molecule of water, thus forming a peptide bond. A polypeptide is a peptide formed by the union of an infinitely (usually large) number of amino acids. Peptides are able to cross the BBB through various mechanisms providing new diagnostic and therapeutic avenues. Various mechanisms are under study. To help in this effort, the "brainpeps" database has been developed. It includes transport information, prioritization of peptide choices for evaluating different $\mathrm{BBB}$ responses, study of quantitative BBB behavior, etc. For example, casomorphion (a heptapeptide) is able to pass the BBB $[15,16]$. 
c. Hexa Decyl Cyano Acrylate (HDCA): Although these have been used against sarcomas in rats, they are not yet ready for clinical trials because the NPs accumulate in the surrounding healthy tissue;

d. PolyAlkylCyanoAcrylate (PACA);

e. The more promising Poly Lactic Glycolic Acid (PLGA) coated with polysorbate 80 or poloxamer 188. Loaded with Doxorubicin, it can be employed in the treatment of glioblastomas (phase I), and

\section{f. Magneto-Electric Nanoparticles (MENs).}

It is clear that NP-based delivery enables sophisticated tactics to fight disease. With their small size and their intricate engineering design, NPs can improve control over drug release profiles, both spatially as well as temporally, and can reduce harmful side effects.

\section{B. Engineered nanoscale devices}

Nanoscale devices can be engineered to aid the delivery of life-saving drug treatments (including cancer) at the affected sites. Such minute devices have the potential to be engineered to efficiently and more safely deliver drug treatments directly to the location of diseased cells while helping avoid harm to healthy cells that fall victim to toxic drugs administered by conventional means. Engineered NDs include:

I. Improved pharmacokinetic strategies of drug molecules (biodistribution, bioavailability, controlled and sitespecific drug release).

\section{Decreased peripheral toxicity.}

III. Influenced manufacturing factors (type of polymers and surfactants, particle size and size distribution, drug molecules), and

IV. Limitations of drug amount delivered, and physiological factors [phagocytic activity of the reticulo-endothelial system (RES), protein opsonization].

\section{Miniaturized drug delivery systems}

Several "nano-carriages" for drug delivery to the right address have been created but many challenges remain, chief among them being how not to let the medicine act before it gets to the right place in the body. The carriers usually encapsulate drugs through long-range electrostatic interactions wherein the carrier attracts oppositely charged medicine. Other tools are available to trigger the release of drugs, for example, an external magnetic field, different $\mathrm{pH}$ values, etc. Such systems, loaded with life-saving drugs, may revolutionize the way in which cancer is treated with chemotherapy, reducing the debilitating side effects of the therapy, making medications more effective, and all the while preserving the healthy living cells. These include:

i. Protein Cages (containing the anticancer drug daunomycin, a small amount of acid and set at a pH below neutral), which slightly open to let the drug jump inside the tumor, stay in until it came in contact with cancer cells. They can kill more than $70 \%$ without attacking healthy cells.

ii. Microbubbles (microscopic balls of gas enclosed in an ultra-thin layer of fat which can be injected into the blood stream). Theoretically, upon reaching the unhealthy part of the body, they are burst with ultrasound waves, releasing the drug exactly where it is needed. Because the entire blood stream is not being flooded with the drug, side-effects from chemotherapy can be greatly reduced, and

iii. Multi-shell hollow nanogels with responsive shell permeability described above.

There are several clinical advantages to these NPs. Specifically, they:

a) Circulate throughout the bloodstream without being attacked by the immune system.

b) Preferentially bind to cancerous areas allowing them to deliver and release their drug payloads specifically there.

c) Are non-toxic as the platelet membranes are nanoparticle cores made of a biodegradable polymer that can be safely metabolized by the body; and

d) Can be packed with many small drug molecules that diffuse out of the polymer core and through the platelet membrane onto their targets.

\section{On The Treatment Of Glioblastomas}

The several FDA-approved drugs for treatment of glioblastomas (GBMs) have had little impact so far because the BBB limits their accumulation. Available drugs cross the BBB in special liposomes (size: $110 \mathrm{~nm}$ ). A potential solution is represented by 3-helix micelles (H3M), a new family of nanocarriers [17-20] consisting of a spherical assembly of ampiphilic hydrophilic and lipophilic peptides and polymers. Micelles are elongated self-assemblies of peptides and polymers, sub(light)microscopic particles $(\sim 20 \mathrm{~nm})$ detected by hydrogels, of supramolecular character and crystalline structure. More recently, they have been defined as one of two classes of colloidal particle, consisting.

a. Of many molecules, the other class being

b. Single macromolecules light- or submicroscopic in size. A micelle is thus a structural unit of the disperse phase in a gel, a unit whose repetition in 3 dimensions constitutes the micellar structure of the gel; it does not denote the individual particles in free suspension or solution, or the unit structure of a crystal. They can be detected by hydrogels of supramolecular character and crystalline structure. H3Ms cross the BBB and accumulate inside GBM tumors at nearly twice the concentration rate of current FDA-approved nanocarriers. They have shown good attributes: long circulation, deep tumor penetration, low accumulation in off-target organs (such as the liver and spleen). They open 
the possibility of treating GBMs via intravenous (IV) [21] drug administration rather than employing invasive measures. Figure 9 illustrates new H3M nanocarriers for effective delivery of new therapeutic drugs to brain tumors. H3M meet all the size and stability requirements for effectively delivering drugs to glioblastoma tumors. They have been demonstrated in rats using radiolabeling with copper (Cu-64) in combination with MRI and PET.

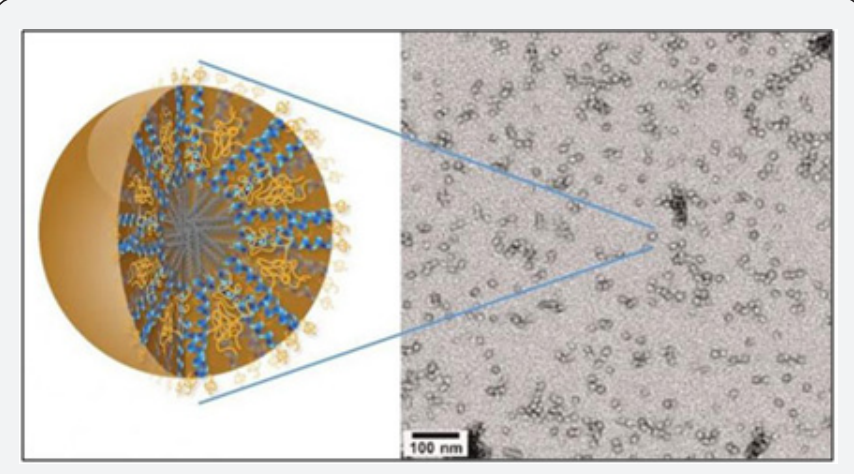

Figure 9: New 3HM nanocarriers for effectively delivery of therapeutic drugs to brain cancer tumors (From: Dr. Ting $\mathrm{Hu}$, Lawrence Berkeley National Laboratory).

\section{Future Prospects}

NT will reduce the need for invasive surgery. However, some devices (implanted catheters and reservoirs) will likely still be needed. Nanomaterials are improving the safety/efficacy of NDs. Nano-engineered probes can deliver drugs at the cellular level using nanofluidic channels. Microchips and biodegradable polymeric NP carriers may be more effective therapeutically for brain tumors. The next-generations: H3M micelles, nanoparticle nasal spray, "sticky" NPs will further these advances and benefits.

In the NP nasal spray, gold NPs of controlled size shape (spherical) and surface charge contain various medicines. Also, ultrasound is used to target a more precise dose to specific areas. The movement of NPs can be tracked by fluorescent tagging [2123]. The first experiments used locusts. In that case, NPs passing through the BBB reached the brain and suffused in a matter of minutes. However, as of this writing, there is as yet no approved NT-based CNS drug that incorporates nanobiotechnology. Nonetheless, while the safety of NPs must be satisfactorily resolved before human use, the future of nanobiotechnology in brain treatment is promising!

\section{References}

1. Fymat AL "Nanoneurology: Drug Delivery Across the Brain Protective Barriers, J Nanomed Res 2017, 5(1):1-4, 00105.

2. Greig NH (1989) "Drug delivery to the brain by blood-brain barrier circumvention and drug modification. Implications of the Blood-Brain Barrier and its Manipulation", in Newwelt EA, Plenum Press, New York 1: 311-67.

3. Smith QR (1993) "Drug delivery to the brain and the role of carrier mediated transport" in Frontiers in Cerebral Vascular Biology: Transport and Its Regulation, Drewes LR and Betz AL, Plenum Press,
USA 83-93.

4. Fromm MF (2000) "P-glycoprotein: A defense mechanism limiting oral bioavailability and CNS accumulation of drugs", Int J Clin Pharmacol. Ther 38(2): 69-74.

5. Rapoport SI, Ohno K, Fredericks WR and Pettigrew KD (1978) "Regional cerebrovascular permeability to [14C] sucrose after osmotic opening of the blood-brain barrier", Brain Res 150(3)653-657.

6. Sanovich E, Bartus RT, Fride PM, Dean RL (1995) "Pathway across blood-brain barrier opened by the bradykinin agonist, RMP-7", Brain Res 705(2): 125-35.

7. Kreuter J (1996) "Nanoparticles and microparticles for drug and vaccine delivery", J Anat 189(3): 503-505.

8. Kreuter J (1994) "Nanoparticles" in Encyclopedia of Pharmaceutical Technology, Marcel Dekker, USA 165-90.

9. Kreuter J (1990) "Large scale production problems and manufacturing of nanoparticles" in Specialized Drug Delivery Systems, Marcel Dekker, USA 257-266.

10. Kreuter J (2001) "Nanoparticulate systems for brain delivery of drugs", Adv Drug Del Rev 47(3): 65-81.

11. Marty JJ, Oppenheim RC, Speiser PP (1978) "Nanoparticles-A new colloidal drug delivery system", Pharm Acta Helva 53(1): 17-23.

12. Muller RH, Mehnert W, Lucks JS (1995) "Solid lipid nanoparticles-An alternative colloidal carrier for controlled drug delivery", Eur J Pharm Biopharm 41: 62-9.

13. Schwarz C, Mehnert W and Lucks JS (1994) "Solid lipid nanoparticles for controlled drug delivery: Production, characterization and sterilization", J Cont Rel 30: 83-96.

14. Schroder U, Sommerfeld P, Ulrich S, Sabel BA (1998) "Nanoparticle technology for delivery of drugs across the blood-brain barrier", J Pharm Sci 87(3: 1305-1307.

15. Schroder U, Sabel BA (1996) "Nanoparticles, a drug carrier system to pass the blood-brain barrier, permit central analgesic effects of dalagrin injections", Brain Res 710: 121-124.

16. Kreuter J, Alyautidin R, Kharkevich DA, Ivanov AA (1995) "Passage of peptides through the blood-brain barrier with colloidal particles (nanoparticles)", Brain Res 674(1): 171-174.

17. Birrenbach G, Speiser PP (1976) “Polymerized micelles and their use as adjuvants in immunology", J. Pharm. Sci 65(12): 1763-1766.

18. TakadaY, Vistica DT, Grei NH, Purdon D, Rapoport SI (1992) “Rapid high affinity transport of a chemotherapeutic amino acid across the bloodbrain barrier", Cancer Res. 52(8): 2191-2196.

19. Gelprina SE, Smirnova ZS, Khalansky AS (2000) "Chemotherapy of brain tumors using doxorubicin bound to polysorbate-80 coated nanoparticles" in Proceedings of the 3rd World Meeting Berlin 441442.

20. Gulyaev AE, Gelperina SE, Skidan IN, Antropov AS (1999) "Significant transport of doxorubicin into the brain with polysorbate-80 coated nanoparticles", Pharm Res 16(10):1564-1569.

21. Gupta PK and Hung CT (1990) "Targeted delivery of low dose doxorubicin hydrochloride administered via magnetic albumin microspheres in rats", J Microencaps 7(1): 85-94.

22. Gupta PK, HungCT, Perrier DG (1986) "Quantitation of the release of doxorubicin from colloidal drug forms using dynamic dialysis", Int J Pharm 33: 137-46.

23. Yang CS, Lu FL, Cai Y (1999) “Body distribution in mice of intravenously injected camphotothericin solid lipid nanoparticles and targeting effect on the brain", J Cont Rel 59: 299-307. 

(C) Commons Attribution 4.0 Licens

DOI: 10.19080/OAJS.2017.04.555654

\section{Your next submission with Juniper Publishers will reach you the below assets}

- Quality Editorial service

- Swift Peer Review

- Reprints availability

- E-prints Service

- Manuscript Podcast for convenient understanding

- Global attainment for your research

- Manuscript accessibility in different formats

( Pdf, E-pub, Full Text, Audio)

- Unceasing customer service

Track the below URL for one-step submission https://juniperpublishers.com/online-submission.php 